\title{
FISIOPATOLOGIA DA HIPOTENSÃO POSTURAL E INTOLERÂNCIA ORTOSTÁTICA
}

\section{PATHOPHYSIOLOGY OF POSTURAL HYPOTENSION AND ORTHOSTATIC INTOLERANCE}

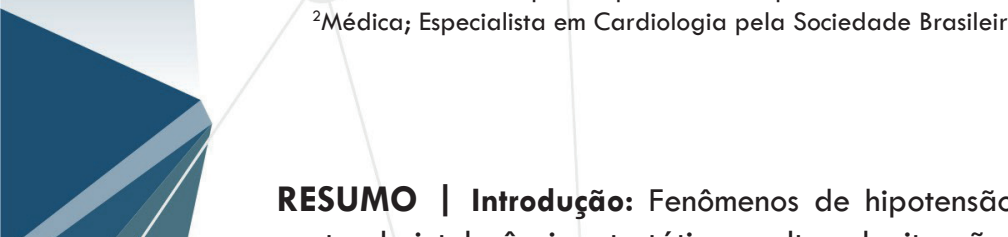 postural e intolerância ortostática resultam de situações que envolvem hipofluxo cerebral transitório, sendo normalmente relacionadas a uma condição benigna e passível de tratamento, seja ele farmacológico ou não. Objetivo: Discorrer sobre os aspectos fisiopatológicos envolvidos nos episódios de perda de consciência relacionados ao sistema cardiovascular. Metodologia: Foi desenvolvida uma revisão de literatura contendo artigos em língua inglesa e portuguesa e capítulos de livros que abordassem aspectos fisiopatológicos na hipotensão postural ou na intolerância ortostática. Resultados/Discussão: Nota-se que os casos benignos de síncope associados ao sistema cardiovascular, principalmente os recorrentes, possuem fisiopatologia complexa, sendo necessária a compreensão dos mecanismos envolvidos aos episódios de perda de consciência para que assim desenvolvam-se melhores maneiras, farmacológicas ou não, de se abordar os doentes envolvidos. Situações com desregulação autonômica estão invariavelmente presentes na gênese dos episódios sincopais. Considerações finais: A fisiopatologia relacionada nos episódios de perda de consciência associados ao sistema cardiovascular envolve fatores como doenças preexistentes, uso de fármacos, envelhecimento, intensidade do exercício e outros, que devem ser considerados pelo profissional de saúde envolvido no processo de assistência a essa população.}

Palavras-chave: Hipotensão Ortostática; Síncope Vasovagal; Seio Carotídeo; Intolerância Ortostática.

\begin{abstract}
Introduction: Phenomena of postural hypotension and orthostatic intolerance result from situations involving transient cerebral hypoflow and are usually related to a benign and amenable condition, whether pharmacological or not. Objective: To discuss the physiopathological aspects involved in the episodes of loss of consciousness related to the cardiovascular system. Methodology: A literature review was developed containing articles in English and Portuguese and chapters of books that address pathophysiological aspects in postural hypotension or orthostatic intolerance. Results / Discussion: Benign cases of syncope associated with the cardiovascular system, mainly recurrent ones, have complex pathophysiology, and it is necessary to understand the mechanisms involved in the episodes of loss of consciousness in order to develop better pharmacological or Not to approach the patients involved. Situations with autonomic dysregulation are invariably present in the genesis of syncopal episodes. Final considerations: The pathophysiology related to the loss of consciousness associated with the cardiovascular system involves factors such as preexisting diseases, drug use, aging, exercise intensity and others, which should be considered by the healthcare professional involved in the care process for this population.
\end{abstract}

Key words: Orthostatic hypotension; Vasovagal Syncope; Carotid sinus; Orthostatic Intolerance. 


\section{INTRODUÇÃO}

Conhecer os fenômenos de hipotensão postural e intolerância ortostática é fundamental para que - profissional de saúde possa ter capacidade para atuar junto a eventos que ocorrem com certa frequência em indivíduos normais e ainda em indivíduos portadores de doença cardiovascular.

Ambos os fenômenos resultam invariavelmente no evento síncope, que por sua vez é definida como uma perda transitória e autolimitada da consciência, seguida de recuperação espontânea sem intervenção terapêutica, e habitualmente acompanhada de perda do tônus postural' .

O termo síncope provém do grego "syncopa". Hipócrates, mil anos antes de Cristo, relatou que pacientes que sofriam de frequentes desmaios geralmente faleciam, enquanto no relato de Engel a única diferença entre síncope e morte súbita seria - fato de que, na primeira hipótese, o paciente acorda'.

Segundo o estudo de Framingham, baseado no seguimento de 7.814 indivíduos por 17 anos, a incidência de síncope foi de 6,2/1.000 pessoas/ ano. A etiologia mais freqüente foi a neuromediada, correspondendo a $21,2 \%$ dos casos $^{2}$.

A assistência aos indivíduos que apresentam de síncope benigna não é uniforme em nosso país. É opinião dos autores que a grande maioria dos cursos de formação, considerando os diversos profissionais de saúde, não aborda profundamente os mecanismos envolvidos na gênese dos episódios de perda de consciência. Compreender os aspectos fisiopatológicos relacionados à hipotensão postural e à intolerância ortostática é fundamental para que as condutas terapêuticas devidas possam ser empregadas devidamente. Essa atualização, que tem por objetivo discorrer sobre os aspectos fisiopatológicos envolvidos nos episódios de perda de consciência relacionados ao sistema cardiovascular.

\section{METODOLOGIA}

Esta revisão de literatura foi conduzida utilizando referências publicadas a partir do ano de 1977, considerando os artigos científicos e a partir de 1983 considerando os livros escolhidos para inclusão. Os artigos selecionados foram escritos em inglês ou português. Os descritores usados para a busca foram Hipotensão Ortostática; Síncope Vasovagal; Seio Carotídeo; Intolerância Ortostática e seus equivalentes em inglês. Realizouse um artigo de revisão de literatura atual, que incluísse revisões (sistemáticas ou não) publicadas por grandes especialistas na área e ainda ensaios clínicos, randomizados ou não, capazes de refletir a melhor evidência disponível na literatura sobre o tema abordado, especificamente sobre os aspectos fisiopatológicos envolvidos nos episódios de hipotensão postural e/ou à intolerância ortostática. Capítulos de livros relevantes para o tema também foram incluídos, sendo os livros provenientes da Biblioteca do Centro de Estudos do Hospital e Maternidade São Cristóvão, em São Paulo/SP.

Ainda no que tange ao detalhamento da metodologia para preparo dessa atualização, buscou-se adotar os seguintes passos: a) Identificação de tópicos a serem atualizados em cada assunto; b) Escolha das palavras-chave; c) Revisão da literatura (base de dados: MEDLINE, LILACS e PubMed); d) Descrição das características da busca. Como estratégia de busca usou-se a descrita no artigo "Efficient literature searching: a core skill for the practice of evidence-based medicine" 3 . A seleção dos estudos foi baseada na leitura do título e do resumo e, quando necessário, na leitura do texto completo por ambos os autores. A revisão bibliográfica se encerrou em 30 de novembro de 2016. Ressaltase que as referências mais antigas foram incluídas pela importância histórica das mesmas no assunto em questão. 


\section{RESULTADOS/DISCUSSÃO}

Hipotensão postural

A definição de hipotensão postural (ortostática) clássica é, ao assumir a posição ortostática (nos três primeiros minutos), ocorrência de queda de, pelo menos, $20 \mathrm{mmHg}$ na pressão arterial sistólica, ou para menos de $90 \mathrm{mmHg}$, ou queda igual ou maior do que $10 \mathrm{mmHg}$ na pressão arterial diastólica ${ }^{4}$. Sinais e sintomas tais como: quedas, tonturas referidas pelo paciente como não rotacionais, présíncopes ou síncopes francas, estão frequentemente presentes nos episódios de hipotensão postural. O evento de hipotensão postural pode estar associado a traumatismo craniano, fraturas em geral e necessidade de hospitalização. Idosos institucionalizados são mais susceptíveis a esse tipo de evento,com até $70 \%$ de prevalência. Idosos na comunidade por sua vez apresentam taxas de eventos que variam entre cinco e $55 \%{ }^{5}$.

Intolerância ortostática

A intolerância ortostática pode se apresentar de diversas formas, ocorrendo a qualquer momento ao se assumir a posição de ortostase. A intolerância ortostática é associada a disfunções do sistema nervoso autônomo. As principais alterações autonômicas que cursam com intolerância ortostática são: síncopes neuromediadas; falências autonômicas puras, sejam elas águas ou crônicas, primárias ou secundárias; atrofias sistêmicas múltiplas como as presentes na Síndrome de Shy-Drager; e ainda as síndromes posturais ortostáticas taquicardizantes (SPOT)'.

O hipofluxo cerebral transitório leva à perda de consciência, que normalmente é benigna e passível de tratamento, seja esse farmacológico ou não². Quedas no fluxo sanguíneo cerebral ao redor de seis segundos estão relacionadas à perda de consciência. Qualquer fator que diminua o débito cardíaco ou a resistência vascular periférica (consequentemente comprometendo a pressão arterial sistêmica) resulta em quedas da perfusão encefálica. Importante ressaltar que nas neuropatias autonômicas, o principal mecanismo envolvido na queda do fluxo sanguíneo cerebral está relacionado à diminuição significante da resistência vascular sistêmica ${ }^{4}$.
A faixa etária interfere na ocorrência das diversas etiologias. As síndromes neuromediadas, psiquiátricas e arritmias primárias são mais frequentes em crianças e jovens. Em adultos as síncopes neuromediadas são mais prevalentes, assim como a perda de consciência relacionada a situações como micção, defecação, visão de sangue e outras. Situações que resultam em obstruções do débito cardíaco, como a estenose aórtica ou a embolia pulmonar são as mais frequentes na intolerância ortostática de indivíduos idosos, que também apresentam alta prevalência de arritmias decorrentes de doença estrutural do coração como causa de síncope. Como idosos apresentam também diversas comorbidades, é frequente o uso de múltiplos medicamentos (como os vasodilatadores e betabloqueadores), que por vezes fazem com que ocorra uma menor capacidade de compensação, quando há variações para menos nos níveis pressóricos ${ }^{4}$.

Ao deparar-se com indivíduos que apresentam recorrência de síncopes ou pré-sincopes recorrentes, - profissional assistente deve sempre investigar as potenciais causas de disfunção autonômica, como medicamentos em uso ou doenças associadas como demências, Mal de Alzheimer, doença de Parkinson, disfunção cerebelar, esclerose múltipla, estados infecciosos, neuropatia diabética, insuficiência renal crônica, síndrome da imunodeficiência adquirida, insuficiência adrenal/glandular, diversos tipos de câncer e ainda alcoolismo'.

Tilt table test

O teste de inclinação passiva (tilt table test) constitui hoje o teste mais importante para o diagnóstico das respostas circulatórias frente ao estresse postural. Sua compreensão é fundamental para que o tratamento do indivíduo acometido pelos fenômenos de hipofluxo cerebral secundários ao ortostatismo. Manter inclinado um paciente em uma mesa de "tilt" é uma maneira de testar a predisposição à perda de consciência. Assume-se que a ocorrência de hipotensão e/ou bradicardia durante a fase de inclinação são equivalentes um evento de síncope espontâneo ${ }^{4,6}$.

O teste de inclinação é realizado com o indivíduo em jejum mínimo de seis horas, sendo então posicionado em decúbito dorsal horizontal sobre uma maca motorizada basculante com suporte para 
os pés e atado à mesma através de dois cintos de segurança. Regularmente realiza-se a monitorização contínua e não invasiva da pressão arterial (com pletismógrafo digital ou tonometria de pulso), além do eletrocardiograma convencional. A intensidade da luz deve estar diminuída durante todo o período de monitorização. Os pacientes permanecem em repouso por 10 a 20 minutos em decúbito dorsal horizontal para medida dos parâmetros basais, com ○ mínimo de estímulos ambientais. A maca é então inclinada em menos de 10 segundos, sendo mantida a 60 graus de inclinação, com o paciente em postura ortostática. $O$ teste dura normalmente 40 minutos ou é interrompida imediatamente, em casos de sintomas de perda de consciência, quando reposiciona-se a maca na horizontal ou ainda em posição de Trendelemburg, quando necessário.

O exame permite a associação de alguns medicamentos (isoproterenol, nitratos ou adenosina) que predispõem os pacientes ao evento sincopal. Tal associação de fármacos ao exame não compromete a especificidade do teste.

Fisiologicamente, ao se realizar a inclinação ortostática passiva do paciente, ocorre o sequestro de sangue para os membros inferiores, o que provoca estímulos aos barorreceptores e aumento da atividade nervosa simpática com o objetivo de aumentar o débito cardíaco. Indivíduos suceptíveis desencadeiam $\circ$ reflexo de Bezold-Jarisch de forma inadequada, com hipotensão e bradicardia paradoxais, que podem resultar em hipoperfusão encefálica ${ }^{6,7}$.

Classifica-se a resposta positiva ao teste de inclinação em subgrupos que levam em consideração as alterações pressóricas e de frequência cardíaca observadas durante a exposição ao decúbito ortostático (quadro 01 ).

Quadro 01. Respostas possíveis ao teste de inclinação $0^{5,6}$

$\begin{array}{ll}\text { Vasodepressora } & \begin{array}{l}\text { queda da pressão arterial sistólica maior ou igual a } 30 \mathrm{mmHg}, \mathrm{sem} \\ \text { alteração significativa da frequência cardíaca }\end{array} \\ \text { Cardioinibitória } & \begin{array}{l}\text { pausa sinusal súbita maior ou igual a três segundos acompanhada de } \\ \text { hipotensão arterial }\end{array}\end{array}$

Mista

queda da pressão arterial maior ou igual a $30 \mathrm{mmHg}$ com concomitante queda da frequência cardíaca

Síndrome da taquicardia postural ortostática (SPOT)

Disautonômica

Hipersensibilidade do seio carotídeo
Aumento da freqüência sinusal superior a 30 batimentos por minuto (bpm) em relação à basal, durante os 40 minutos de monitorização em decúbito ortostático, ou o desenvolvimento de taquicardia sinusal maior ou igual a $120 \mathrm{bpm}$ nos primeiros 10 minutos de exposição ao decúbito ortostático, com sintomas de intolerância ortostática

queda gradual e progressiva da pressão arterial durante a exposição ao teste de inclinação, acompanhada ou não de discreto aumento da frequência cardíaca. Normalmente as pressões (sistólica e diastólica) caem em paralelo uma à outra

na ausência de drogas depressoras do nó sinusal e da condução atrioventricular, ocorre pausa ventricular maior que três segundos - por parada sinusal ou bloqueio atrioventricular - e/ou queda da pressão arterial sistólica igual ou maior que $50 \mathrm{mmHg}$, durante manobra de compressão do seio carotídeo por cinco a 10 segundos, realizada nos primeiros minutos de inclinação (normalmente após dois minutos de inclinação) 
Espera-se que indivíduos normais ao serem colocados em pé apresentem um aumento na atividade simpática e redução na atividade parassimpática, com otimização do retorno venoso e do débito cardíaco'. Nos indivíduos acometidos pela síncope neuromediada, essas alterações não ocorrem, sendo observado um desbalanço no sistema nervoso autônomo'. Nem todos os mecanismos fisiopatológicos envolvidos na gênese dos episódios de síncope neuromediada foram desvendados até o momento. Atribui-se a perda de consciência à incapacidade dos mecanismos reflexos compensatórios responsáveis por manter os níveis de pressão arterial (receptores cardiopulmonares e barorreflexo arterial). Na resposta neuromediada, também chamada de vasovagal ocorre bradicardia e vasodilatação arterial em face de uma pressão arterial já descendente, caracterizando portanto a incapacidade dos mecanismos compensatórios que deveriam manter uma pessão arterial adequada. Ocorre aumento da atividade vagal e diminuição da atividade simpática sobre o sistema cardiovascular, sendo o principal estímulo deflagrador para tal a ativação de receptores intracardíacos (mecanorreceptores/fibras C), localizados especialmente na parede ínfero-lateral do ventrículo esquerdo. As fibras $C$ ventriculares são estimuladas em situações em que o retorno venoso se encontra diminuído, como na posição ortostática, ou em situações de hipovolemia, o que provoca contrações cardíacas vigorosas com o coração relativamente "vazio", o que desencadeia aumento da atividade vagal por meiodo reflexo de Bezold-Jarisch, com bradicardia e/ou hipotensão, resultando em perda de consciência ${ }^{2}$.

\section{Síndrome postural ortostática taquicárdica (SPOT)}

Um tipo especial de disfunção autonômica crônica é a síndrome postural ortostática taquicárdica (SPOT). A principal característica desta síndrome é a taquicardia persistente (algumas vezes alcançase 160 batimentos por minuto ou mais) quando o paciente está na posição ortostática, acompanhada de fadiga severa e crônica, intolerância ao exercício, tontura e pré-síncope. Muitos indivíduos portadores se queixam de que estão sempre com frio e, ao mesmo tempo, têm intolerância ao calor extremo. Acredita- se que esta síndrome corresponda a uma forma inicial de disfunção autonômica vascular periférica. O mecanismo subjacente a esta condição parece ser uma insuficiência do sistema vascular periférico de vasoconstrição, que deveria acontecer como uma resposta compensatória à posição ortostática. $\mathrm{Na}$ ausência de vasoconstrição adequada, o organismo acaba por lançar mão do aumento da freqüência cardíaca, que pode alcançar níveis bastante elevados 8 .

Hipersensibilidade do seio carotídeo

Idosos que apresentam sintomas de tonturas, escurecimento visual, pré-síncopes e síncope quando realizam compressões ou manipulações na região do seio carotídeo podem ser acometidos por uma disfunção caracterizada como hipersensibilidade do seio carotídeo. Situações como o ato de barbear-se, uso de colarinhos apertados ou gravatas, rotações laterais ou extensão brusca do pescoço podem desencadear os sinais e sintomas, devido à compressão mecânica do seio carotídeo. Considerando seu diagnóstico, no momento do teste de inclinação realiza-se a compressão leve e rápida (cinco a 10 segundos) na região carotídea, um lado de cada vez. Após 15 segundos pode-se repetir a manobra, com aumento na intensidade da compressão. Considera-se positividade quando ocorrem sintomas que regularmente são associados a pausas sinusais maiores que três segundos e/ou hipotensão (as respostas mais comuns costumam ser pausas sinusais ou bloqueio atrioventricular durante a massagem, com desencadeamento de sintomas). Sopros carotídeos e doença aterosclerótica carotídea relevante contra-indicam o procedimento'.

Ao se realizar o diagnóstico, em casos selecionados (nas formas cardioinibitórias ou mistas) há necessidade de implante de marcapasso cardíaco definitivo, uma vez que a resposta cardioinibitória é muito relevante nas hipersensibilidades de seio carotídeo. O implante de marcapasso não garante remissão completa dos episódios, ocorrendo ainda mais de $10 \%$ de recorrência, em razão do componente vasodepressor'. Para as formas vasodepressoras o tratamento é semelhante ao da síncope neuromediada ${ }^{4}$. A resposta vasodepressora precisa ser aferida por técnicas de medição contínua da pressão, sendo valorizadas quedas de mais de $50 \mathrm{mmHg}$, com sintomas associados'. 
A presença de cirurgias cervicais prévias, câncer cervical ou radioterapia nessa região deve ser pesquisada, pois em casos como esses, pacientes podem ter respostas positivas no teste de compressão do seio carotídeo, sem história clínica de síncopes ou pré-síncopes'.

Síndrome de Shy-Drager e falência autonômica pura

A atrofia sistêmica múltipla, também chamada de Síndrome de Shy-Drager é uma doença progressiva, degenerativa, que apresenta gliose e perda neuronal em várias áreas do sistema nervoso. Tem como sintomas comuns a presença de tonturas, lipotímias ou síncopes, além de frequente hipotensão postural ${ }^{1}$. Essa doença resulta adicionalmente no comprometimento das funções cerebelares (degeneração olivopontocerebelar) ou parkinsonianas (degeneração estritonigral), sendo nessa, predominantes os sintomas de rigidez e bradicinesia, com menos tremores do que comumente visto nos pacientes com doença de Parkinson'. Os pacientes com parkinsonismo não respondem bem a levodopa, sabendo-se que muitos pacientes com o diagnóstico prévio de doença de Parkinson apresentam de fato essa síndrome'.

Falências autonômicas puras são menos frequentes, tendo um melhor prognóstico que a atrofia sistêmica múltipla. Compromete também os sistemas simpático e parassimpático, não tendo alterações centrais, e sim envolvimento dos neurônios pós-ganglionares periféricos. Encontram-se níveis diminuídos de noradrenalina basal nessa disfunção, que não se observa nos pacientes de Shy-Drager'.

Ambos têm respostas atenuadas de aumento da noradrenalina plasmática durante $\circ$ teste de inclinação'.

Causas secundárias de disfunção autonômica

Em alguns pacientes, podem coexistir distintas condições cujo efeito sinérgico leva à disfunção autonômica. Há várias anormalidades enzimáticas que podem causar distúrbio na regulação do sistema nervoso autônomo. Uma dessas anormalidades é a síndrome da deficiência da beta hidroxilase dopamina, condição facilmente tratada com a reposição enzimática.
Ocorrem também síndromes causadas por diminuição ou ausência de substâncias como o fator de crescimento dos nervos, monoaminoxidase; L-aminodecarboxilase aromática; e alguns neuropeptídeos sensoriais. Quando de sua menor quantidade no organismo, eventualmente acontece insuficiência autonômica e hipotensão ${ }^{8}$.

Quando se discute a disfunção autonômica associada à intolerância ortostática ou à hipotensão postural, cabe ressaltar que uma vasta quantidade de medicamentos pode piorar ou causar os eventos. Dentre esses é frequente a associação da ocorrência de pré-síncopes ou síncopes com fármacos como os inibidores da enzima conversora da angiotensina, bloqueadores alfa-adrenérgicos, bloqueadores dos canais de cálcio, betabloqueadores, fenotiazidas, antidepressivos tricíclicos, reserpina, metildopa, bromocriptina, etanol, opiáceos, diuréticos, hidralazina, agentes bloqueadores ganglionares, nitratos, citrato de sildenafila e inibidores da monoaminoxidase ${ }^{8}$.

Pacientes com insuficiência cardíaca congestiva tem muitas vezes um aumento de frequência de síncope por disautonomia. Especificamente, a combinação de débito cardíaco baixo e depleção volumétrica (devido ao uso de diuréticos e terapêutica vasodilatadora) pode interferir nos mecanismos normais de adaptação ao ortostatismo ${ }^{8}$.

Intolerância ortostática e exercício

Durante o exercício, podem ocorrer síncopes neuromediadas pela vasodilatação exacerbada do leito vascular nas áreas utilizadas (músculos) durante a atividade e a não ocorrência de vasoconstrição reflexa das áreas não utilizadas (como as vísceras). Elegante estudo evidenciou que a resistência vascular, medida no antebraço, encontrava-se significativamente menor em pacientes do que em indivíduos controles no pico do exercício que foi realizado com os membros inferiores. Notou-se, no mesmo estudo, que a resistência vascular no antebraço caiu nos pacientes, enquanto aumentou nos controles. Além disso, a pressão sistólica era significantemente menor nos pacientes portadores de síncope durante o exercício?.

Atualmente, mesmo como todo o suporte prestado aos atletas pelas equipes especializadas em Fisiologia 
do Exercício, pode ocorrer desidratação eventual, que costuma acompanhar a realização os exercícios de média ou longa duração. Foi descrita perda de peso de aproximadamente $3,5 \mathrm{~kg}$ e diminuição no volume de plasma de aproximadamente 12,8\% em atletas praticantes de ultramaratona, o que pode predispor à queda de pressão em indivíduos susceptíveis. $O$ seqüestro de sangue para as regiões da pele, que acontece durante o exercício de alta intensidade e/ou longa duração pode também favorecer a hipoperfusão cerebral. Quando uma pessoa está em repouso, cerca de $5 \%$ dos cinco litros de sangue bombeados a cada minuto pelo coração vão para a pele. Isso contrasta com o exercício realizado em um ambiente quente e úmido, quando até $20 \%$ do fluxo sanguíneo total é desviado para a superfície corporal para que se mantenha a termorregulação, considerando os mecanismos de perda de calor $^{10,11}$.

A hiperventilação muitas vezes está presente durante o exercício. Foi descrito que atletas bem treinados submetidos à manobra voluntária de hiperventilação apresentaram diminuição da frequência cardíaca basal e assistolia, tanto durante quanto após a manobra, acompanhadas de síncope e convulsões. Ao ato de hiperventilar atribui-se a ocorrência de queda na concentração sanguínea de dióxido de carbono (alcalose respiratória), aumento da resistência cerebrovascular e diminuição do fluxo sanguíneo cerebral, fatores que poderiam agravar - desencadeamento do episódio em indivíduos susceptíveis. Considerando a assistolia, a mesma pode ter sua origem em dois componentes. Primeiro, uma súbita e momentânea queda na atividade nervosa simpática associada com uma forte e temporária predominância vagal, que ocorreu imediatamente após a manobra de hiperventilação. Segundo, uma resposta vagal exacerbada dos receptores cardiopulmonares, submetidos à estimulação mecânica pelo ato inspiratório' ${ }^{12}$.

Foi demonstrado que a auto-regulação da circulação sanguínea cerebral é essencialmente regulada por um componente metabólico, em lugar de fenômenos miogênicos, mecânicos ou neurovasculares. Durante estresse postural, verificou-se que a resistência vascular periférica sofre aumento, concomitante com ativação do sistema simpático, sem repercussões importantes na circulação sanguínea cerebral em indivíduos normais. Sabe-se que o exercício físico de alta intensidade é acompanhado por intensa ativação simpática e que vasos cerebrais são altamente inervados por fibras adrenérgicas ${ }^{13}$.

Situações de exercício intenso podem desencadear um extremo aumento do o tônus vasoconstritor simpático, que eventualmente pode sobrepujar - estímulo metabólico vasodilatador, gerando diminuição no fluxo sanguíneo cerebral e síncope. A parada súbita no exercício físico, sobretudo após períodos longos de realização, poderia ocasionar hipofluxo na circulação sanguínea cerebral, resultante de altos índices de atividade simpática sobre os vasos cerebrais e súbita queda do débito cardíaco por diminuição do retorno venoso ${ }^{14}$.

Murrell e colaboradores descrevem a ocorrência de hipotensão importante em maratonistas, mesmo com os indivíduos em posição supina, após a realização de uma prova. Os dados adquiridos demonstraram uma significante diminuição da sensibilidade barorreflexa, $\circ$ que poderia predispor esses indivíduos (maratonistas experientes) a fenômenos de perda de consciência, por incapacidade do sistema nervoso simpático em gerar vasoconstrição periférica e uma boa redistribuição do fluxo sanguíneo. Os autores sugerem ainda que a hidratação, realizada durante a maratona, foi efetiva em manter os volumes plasmáticos no término da corrida, mas esse fator não foi suficiente para evitar episódios de síncope e pré-síncope, quando os indivíduos estudados foram solicitados a permanecer em pé por seis minutos, em um período de até duas horas após a prova. A diminuição da sensibilidade barorreflexa nessa população desapareceu após 48 horas do término da corrida, com normalização dos valores de pressão arterial e desaparecimento dos sintomas relacionados à intolerância ortostática ${ }^{15}$.

Indivíduos que se submetam a treinamento físico intenso, com altas cargas de trabalho (situação comum em atletas de alto rendimento), podem se tornar mais predispostos à ocorrência de fenômenos de pré-síncope, síncope ou intolerância ortostática. 2 O componente psicológico precisa ser também avaliado. Foi descrito o caso de uma atleta que sofreu um episódio importante de síncope durante uma prova em de alto rendimento. Ao recobrar os sentidos, a mesma apresentava fraqueza generalizada e parestesias em hemicorpo esquerdo, 
além de visão turva. Depois de ampla investigação, descobriu-se, por meio de acompanhamento psicológico, que a atleta apresentava altos índices de ansiedade. Após reabilitação cardiovascular e psicoterapia, a mesma manifestou estar apta para retornar à prática esportiva em duas semanas após a perda de consciência, com completa remissão dos sintomas ${ }^{16}$.

Intolerância ortostática em portadores de AIDS internados em ambiente hospitalar

A disfunção autonômica é comum em pacientes com HIV/AIDS e tem maior prevalência em pacientes com estágio da doença mais avançado. Esta disfunção autonômica pode ser causada pela própria utilização da terapia de antiretroviral (TARV), que é neurotóxica e possui efeito diabetogênico (leva a um dano parassimpático assim como nos pacientes diabéticos) ${ }^{17}$.

Sabe-se também que a restrição ao leito decorrente da hospitalização, pode fazer com que os indivíduos internados sofram os efeitos do imobilismo, que somados às condições clínicas características do paciente portador de HIV/AIDS (caquexia, infecções oportunistas, doenças neurológicas, fraqueza muscular), poderiam levar esse paciente à deterioração funcional, alterações fisiológicas e perda da independência em atividades cotidianas. As consequências do decúbito prolongado podem ainda levar a alterações cardiocirculatórias e posteriormente alterações hemodinâmicas e, com isto, disfunção ortostática ${ }^{17}$. Em pacientes com estas características notou-se a presença de disfunção autonômica parassimpática (aumento da frequência cardíaca de repouso e diminuição da variabilidade da frequência cardíaca) relacionada a TARV ${ }^{17}$. Importante salientar que indivíduos que apresentem menor exposição ao estresse ortostático, ou seja, gradiente gravitacional reduzido, situação similar à restrição ao leito ou à exposição à microgravidade, apresentam alterações cardiocirculatórias, incluindo hipovolemia, redução da sensibilidade barorreflexa e aumento do acúmulo de sangue nas regiões periféricas do organismo. Essas alterações podem ser consideradas como descondicionamento cardiovascular, o que pode levar à intolerância ortostática.

Pacientes com HIV/AIDS fazem uso associado de fármacos. Entre estes fármacos encontram-se os inibidores de protease (IPs), que podem causar disfunção endotelial, cuja fisiopatologia ainda não está totalmente esclarecida, e diminuição da capacidade vasodilatadora ${ }^{17}$.

Sabe-se que o decúbito horizontal prolongado leva à disfunção barorreflexa, gerando alterações no fluxo sanguíneo ${ }^{17}$. Um estudo realizado no Brasil analisou a tolerância ortostática e a variabilidade da frequência cardíaca (VFC) em indivíduos portadores de HIV/AIDS, internados em enfermaria hospitalar por sete dias, que permaneceram acamados no leito por $19 \pm 2$ horas/dia. Realizouse o teste de inclinação no dia 07 . Como resultado, $33 \%$ da amostra apresentou positividade ao teste, com maior predomínio simpático em repouso e durante a inclinação ortostática, quando comparados a pacientes com teste de inclinação negativo. Os autores sugerem em suas conclusões que $\circ$ componente simpático aumentado no grupo que apresentou a perda de consciência poderia desencadear o reflexo de Bezold-Jarisch, justificando as síncopes ${ }^{17}$.

\section{Diagnóstico}

O diagnóstico da hipotensão postural e da intolerância ortostática é feito, principalmente, a partir da anamnese, associando-se normalmente $\circ$ uso do teste de inclinação ortostática ${ }^{4,18}$. O resultado obtido durante o tilt teste pode ser utilizado como preditor de sucesso do tratamento escolhido para pacientes específicos. Admite-se que a não ocorrência de sintomas ou síncope durante o teste esteja relacionada à efetividade da terapêutica escolhida ${ }^{18}$.

Os episódios de síncope neuromediada são muitas vezes relatados em consultório, sendo que a clínica dos pacientes já auxilia sobremaneira para seu diagnóstico. Regularmente é descrita a frequência de eventos, intensidade e duração das perdas de consciência. Os sintomas pré síncopais podem variar entre diferentes indivíduos, e incluem sensações de sudorese, palpitações, tremores, agitação, "boca seca", tonturas, relaxamento de esfíncteres, vômitos, convulsões e outros. A síncope neuromediada pode também ocorrer sem a presença de sintomas prévios, - que indica uma série de cuidados especiais no momento da escolha do tratamento, considerando a 
maior tendência a traumas físicos nesses indivíduos. Múltiplos episódios de síncope, que se iniciam com períodos prodrômicos longos (náuseas, calor, tonturas e outros) e que ocorrem em situações específicas, como medo, após o esforço físico quando o indivíduo permanece em postura ortostática, acompanhados de fadiga pós-sincopal, também motivam a suspeita de síncopes neuromediadas ${ }^{4,18}$.

\section{CONCLUSÃO}

A fisiopatologia envolvida nos episódios de perda de consciência associados ao sistema cardiovascular é complexa. Fatores como doenças preexistentes, uso de fármacos, envelhecimento, intensidade do exercício e outros devem ser considerados pelo profissional de saúde envolvido no processo de assistência a essa população.

\section{CONTRIBUIÇÕES DOS AUTORES}

Gardenghi $G$ foi responsável pelo levantamento bibliográfico e redação do manuscrito. Balestra LF foi responsável pela revisão crítica do manuscrito.

\section{CONFLITOS DE INTERESSES}

Nenhum conflito financeiro, legal ou político envolvendo terceiros (governo, empresas e fundações privadas, etc.) foi declarado para nenhum aspecto do trabalho submetido (incluindo mas não limitandose a subvenções e financiamentos, conselho consultivo, desenho de estudo, preparação de manuscrito, análise estatística, etc).

\section{REFERÊNCIAS}

1. Rocha EA. Síndromes neuralmente mediadas. Arq Bras Cardiol. 2006;86(3): e34-e44

2. Hachul DT, de Matos LDNJ, Gardenghi G, Scanavacca, MI. Síncope e morte súbita relacionadas ao exercício: aspectos epidemiológicos e clínicos. In: Negrão CE, Barreto ACP, editores. Cardiologia do exercício: do atleta ao cardiopata. $3^{a}$ ed. Barueri (SP): Ed. Manole; 2010. P. 628-648

3. Doig GS, Simpson F. Efficient literature searching: a core skill for the practice of evidence-based medicine. Intensive
Care Med. 2003;29(12):21 19-27. doi: 10.1007/s00134003-1942-5

4. Macatrão-Costa M, Hachul D. Síncope [Internet]. 2009 [acessado em 08 mar 2015]. Disponível em: http://www. medicinanet.com.br/conteudos/revisoes/1441/sincope.htm

5. da Silva RML, Dias FLC, de Moraes EM, Caramelli P. Análise do sistema nervoso autônomo em idosos de acordo com a presença de intolerância ortostática e demência: um estudo pareado. Relampa. 2013;26(1):17-23

6. Gardenghi G, Hachul DT, Negrão CE. Síncope neurocardiogênica e exercício. Relampa. 2004;17(1):3-10

7. Brito Júnior $\mathrm{HL}$, Hachul DT. O teste de inclinação na síncope. Relampa. $2001 ; 14(1)$ : 17-26

8. Grubb BP, Jorge SC. Aspectos da classificação, diagnóstico e tratamento das síndromes de disfunção autonômica associadas à intolerância ortostática. Arq Bras Cardiol. $2000 ; 74(6): 537-544$

9. Thomson HL, Lele SS, Atherton JJ, Wright KN, Stafford W, Frenneaux MP. Abnormal forearm responses during leg exercise in patients with vasovagal syncope. Circulation. $1995 ; 15,92(8): 2204-09$

10. Holtzhausen LM, Noakes TD. The prevalence and significance of post-exercise (postural) hypotension in ultramarathon runners. Med Sci Sports Exercise. 1995;27(12):1595-1601

1 1. McArdle WD, Katch FI, Katch VL. Fisiologia do Exercício. $3^{a}$ edição. Ed. Guanabara-Koogan; 1992

12. Buja G, Folino AF, Bittante $M$, Canciani B, Martini $B$, Miorelli M et al. Asystole with Syncope Secondary to Hyperventilation in Three Young Athletes. Pace. $1989 ; 12: 406-12$

13. Heistad DD, Kontas HÁ. Cerebral Circulation. In JJ Shepperd, FM Abboud (eds.): Handbook of Physiology, section 2: The Cardiovascular System. Bathesda, MD. American Physiological Society. 1983:137-82

14. Secher NH, Clausen JP, Klausen K, Noer I, Trap-Jensen J. Central and Regional Circulatory Effects of Adding Arm Exercise to Leg Exercise. Acta Physiol Scand. 1977;100: 28897. doi: $10.1111 /$ j.1748-1716.1977.tb05952.x

15. Murrell C, Wilon L, Cotter JD, Lucas S, Ogoh S, George $\mathrm{K}$ et al. Alterations in autonomic function and cerebral hemodynamics to orthostatic challenge following a mountain marathon. J Appl Physiol. 2007;103:88-96. doi: 10.1152/ japplphysiol.01396.2006

16. Lively MW. Syncope and neurologic deficits in a track athlete: a case report. Med Sci Sports Exercise. $2001 ; 33(3): 345-47$ 
17. Justino CF, Santos DS, Vicentim TK, Alexandre L, Salomão R, Gardenghi G. Análise da tolerância ortostática e da variabilidade da frequência cardíaca em indivíduos portadores de HIV/AIDS. Rev Soc Cardiol Estado de São Paulo 2010; 20(3 Supl A):26-30

18. Gardenghi G, Balestra LF. Non-pharmacological treatment in neurally mediated syncope. Relampa.

$2011 ; 24(3): 145-150$ 